\title{
AnÁlisis matemático al MÉtodo D'HONDT DE ASIGNACIÓN DE ESCAÑOS PARLAMENTARIOS
}

\author{
Bolívar Enrique Loján Fierro ${ }^{1, *}$
}

\section{Resumen}

El método de D'Hondt, basado en divisiones sucesivas, es utilizado en muchos países para la repartición de escaños al Parlamento. El método presenta dificultades en las asignaciones cuando las cantidades de votos tienen relaciones, entre sí, de $1,1 / 2,1 / 3, \ldots, 1 / n$, o 1,2 , $3, \ldots, n$ donde $n$ es el número máximo de escaños que se disputan. Se demuestra con teorías de continuidad de funciones, geometría analítica y ejemplos, los casos de discontinuidad que afectan de manera importante al método. Estas situaciones se dilucidan a través de sorteos en casos de empate en la asignación de escaños.

Palabras clave: inconsistencias método de D'Hondt, derechos civiles D'Hondt, base matemática método D'Hondt, repartición de escaños Ecuador.

\begin{abstract}
The D'Hondt method, based on successive divisions, isused in many countries for the distribution of seats in Parliament. The method presents difficulties in assignments where the quantities of votes have relationships among themselves, $1,1 / 2,1 / 3, \ldots, 1 / n$, or $1,2,3, \ldots$, $n$ where $n$ is the maximum number of vyingseats. It is demonstrated with theory of continuous functions, analytic geometry, and examples, that the cases of discontinuity significantly affect the model. These situations are elucidated through raffles in cases of a tie when allocation of seats.
\end{abstract}

Keywords: D'Hondt method inconsistencies, D'Hondt civil rights, basic math D'Hondt method, distribution of seats.

${ }_{1, *}$ Especialista Sistemas de Información, Ingeniero Electricista, Licenciado en Contaduría, Profesor titular en Sistemas, Universidad de Oriente - Venezuela. Autor para correspondencia: portatil@inprocesa.com

Recibido: 14 - Noviembre - 2012, Aprobado tras revisión: 25 - Noviembre - 2012

Forma sugerida de citación: Loján, B. (2012). "Análisis matemático al Método D’Hondt de asignación de escaños parlamentarios". INGENIUS. N. ${ }^{\circ} 8$, (Julio/Diciembre). pp. 30-37. ISSN: 1390-650X 


\section{Introducción}

Las elecciones parlamentarias tienen como fin llevar representantes del elector a ocupar escaños en el Parlamento. Es necesaria entonces la escogencia de un método para hacer las asignaciones de escaños en función de los votos válidos obtenidos por circunscripción electoral. En el caso específico de la República de Ecuador para las elecciones del 2013 [1], se escogió el método de D'Hondt [2], para la asignación de $n$ escaños, que se basa en divisiones sucesivas entre 1 , $2,3 \ldots n$, de los votos obtenidos, como lo muestra la Tabla 1.

Tabla 1. Asignación de cuatro escaños (los asignados están en negrita).

\begin{tabular}{|c|cccc|}
\hline 4 & & Partido A & Partido B & Partido C \\
\cline { 2 - 5 } 5 & Votos & 45000,00 & 23000,00 & 0,00 \\
6 & Asambleístas & & & \\
7 & 1 & $\mathbf{4 5 0 0 0 , 0 0}$ & 23000,00 & 0,00 \\
8 & 1 & 22500,00 & $\mathbf{2 3 0 0 0 , 0 0}$ & 0,00 \\
9 & 1 & $\mathbf{2 2 5 0 0 , 0 0}$ & 11500,00 & 0,00 \\
10 & 1 & $\mathbf{1 5 0 0 0 , 0 0}$ & 11500,00 & 0,00 \\
\hline
\end{tabular}

\subsection{Algoritmo de asignación o cómo funciona el método}

Paso 1:

Se comparan los resultados de la fila 7 , de la Tabla 1 , y se asigna el primer escaño a quien tenga el máximo, en este caso 45000.

Paso 2:

Se divide el total del beneficiado (45000) entre 2, se compara el valor resultante con el total obtenido por la organización B y se asigna el segundo escaño a quien tenga el máximo de la fila 8 en la Tabla 1.

Paso 3:

Se divide el total del beneficiado (23000) entre 2 (fila 9 de la Tabla 1) y se asigna el tercer escaño a quien tenga el máximo.

Paso 4

Se divide el total del beneficiado (45000) entre 3 y se asigna el escaño a quien tenga el máximo de la fila 10 de la Tabla 1.

Se generan tantas series de divisores como ganadores de por lo menos un escaño existan. La serie del último beneficiado, se incrementa en una unidad, hasta completar $n$ escaños o llegar a un sorteo. En la Tabla 2 se muestra la asignación de cinco escaños entre cuatro organizaciones.

El caso de reparto de seis escaños entre tres organizaciones políticas con el problema final de dos escaños
Tabla 2. Asignación de cinco escaños entre cuatro organizaciones políticas.

\begin{tabular}{|c|ccccc|}
\hline 4 & & Partido A & Partido B & Partido C & Partido D \\
\cline { 2 - 5 } 5 & Votos & 45000,00 & 23000,00 & 21000,00 & 35000,00 \\
6 & Asambleístas & & & & \\
7 & 1 & $\mathbf{4 5 0 0 0 , 0 0}$ & 23000,00 & 21000,00 & 35000,00 \\
8 & 1 & 22500,00 & 23000,00 & 21000,00 & $\mathbf{3 5 0 0 0 , 0 0}$ \\
9 & 1 & 22500,00 & $\mathbf{2 3 0 0 0 , 0 0}$ & 21000,00 & 17500,00 \\
10 & 1 & $\mathbf{2 2 5 0 0 , 0 0}$ & 11500,00 & 21000,00 & 17500,00 \\
11 & 1 & 15000,00 & 11500,00 & $\mathbf{2 1 0 0 0 , 0 0}$ & 17500,00 \\
\hline
\end{tabular}

restantes a ser repartidos entre los tres aspirantes se ilustra en la Tabla 3 en la última fila de asignación. Situación que se resuelve con un sorteo.

Tabla 3. Asignación de dos asambleístas entre tres aspirantes de la última fila que se resuelve con sorteo.

\begin{tabular}{|ccc|}
\hline 24,00 & $\mathbf{1 2 0 , 0 0}$ & 24,00 \\
24,00 & $\mathbf{6 0 , 0 0}$ & 24,00 \\
24,00 & $\mathbf{4 0 , 0 0}$ & 24,00 \\
24,00 & $\mathbf{3 0 , 0 0}$ & 24,00 \\
$\mathbf{2 4 , 0 0}$ & $\mathbf{2 4 , 0 0}$ & $\mathbf{2 4 , 0 0}$ \\
\multicolumn{4}{|c}{ Escaños a repartir: 6} \\
\hline
\end{tabular}

En la Tabla 4 se muestran dos organizaciones con igual cantidad de votos que aspiran a tres escaños. En la primera asignación (fila 7) hay dos escaños para dos aspirantes y en la siguiente (fila 8) hay un escaño para dos aspirantes, situación que se resuelve con un sorteo.

Tabla 4. Asignación de un escaño para dos organizaciones con sorteo (fila 8).

\begin{tabular}{|l|cccc|}
\hline 4 & & Partido A & Partido B & Partido C \\
\cline { 2 - 5 } 5 & Votos & 45000,00 & 45000,00 & 0,00 \\
6 & Asambleístas & & & \\
7 & 2 & $\mathbf{4 5 0 0 0 , 0 0}$ & $\mathbf{4 5 0 0 0 , 0 0}$ & 0,00 \\
8 & 2 & $\mathbf{2 2 5 0 0 , 0 0}$ & $\mathbf{2 2 5 0 0 , 0 0}$ & 0,00 \\
\hline
\end{tabular}

El caso de repartición de un número impar de escaños entre un número par de organizaciones con igual número de votos siempre terminará en un sorteo.

\section{Revisión teórica}

La primera acción fue la de conocer la base teórica del método [3] y crear un simulador [4] para comprobar la existencia de puntos sensibles. Este simulador y la geometría analítica coadyuvaron a determinar los posibles puntos que generan situación de sorteo para la asignación de escaños.

El método asigna los escaños sin recurrir al sorteo mientras se satisfagan las condiciones siguientes: 


\subsection{Primera exigencia}

Que los escaños por repartir o restantes sean mayores o iguales a la cantidad de organizaciones con igual cantidad de votos en la fila corriente.

\subsection{Segunda exigencia, para dos escaños y dos organizaciones}

Que entre un par de totales no haya relaciones tipo $1 / k$ o $k(1<k \leq n$ número de escaños).

$$
\begin{gathered}
1 / k=\frac{1}{2} \\
k=2
\end{gathered}
$$

Estas relaciones representan dos rectas de pendiente $\frac{1}{2}$ y 2 (simétricas con respecto a la recta de la identidad) como muestra la Figura 1 con sus posibles escenarios. La región está limitada por la recta frontera determinada por la Ecuación 3.

Votos org. $A+$ Votos org. $B=$

Total votos válidos sufragados

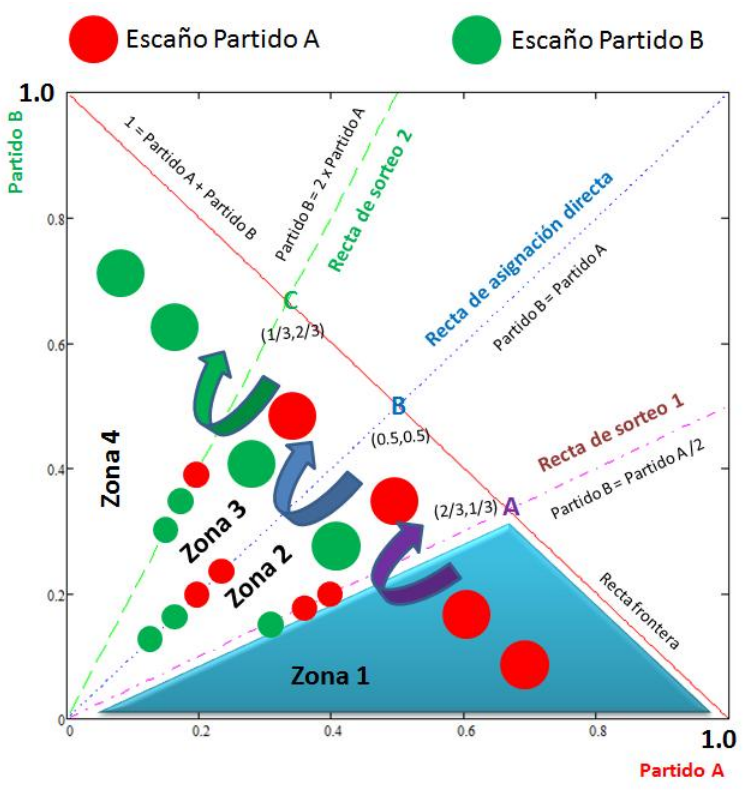

Figura 1. Zonas de asignación de 2 escaños a 2 organizaciones.

$$
\begin{aligned}
& \text { Votos org. } B=\frac{1}{2} \cdot \text { Votos org. } A \\
& \text { Votos org. } B=2 \cdot \text { Votos org. } A
\end{aligned}
$$

Toda combinación de votos que se encuentre sobre las rectas de Ecuaciones 4 y 5 tiene que recurrir al sorteo para dirimir el último escaño, como se observa en las Tablas 5 y 6 .

Para tres organizaciones es necesario plantearse escenarios en tres dimensiones $(3 D)$.

Tabla 5. Asignación dos escaños a dos organizaciones con relación de votos $=1 / 2$.

\begin{tabular}{|c|cccc|}
\hline 4 & & Partido A & Partido B & Partido C \\
\cline { 2 - 5 } 5 & Votos & 200,00 & 100,00 & 0,00 \\
6 & Asambleístas & & & \\
7 & 1 & $\mathbf{2 0 0 , 0 0}$ & 100,00 & 0,00 \\
8 & 2 & $\mathbf{1 0 0 , 0 0}$ & $\mathbf{1 0 0 , 0 0}$ & 0,00 \\
\hline
\end{tabular}

Tabla 6. Asignación dos escaños a dos organizaciones con relación de votos $=2 / 1$.

\begin{tabular}{|l|cccc|}
\hline 4 & & Partido A & Partido B & Partido C \\
\cline { 2 - 5 } 5 & Votos & 100,00 & 200,00 & 0,00 \\
6 & Asambleístas & & & \\
7 & 1 & 100,00 & $\mathbf{2 0 0 , 0 0}$ & 0,00 \\
8 & 2 & $\mathbf{1 0 0 , 0 0}$ & $\mathbf{1 0 0 , 0 0}$ & 0,00 \\
\hline
\end{tabular}

Los elementos contemplados en las Figuras 1 y 2 determinan la discontinuidad de la función de asignación para el caso especial de dos escaños y dos organizaciones competidoras.

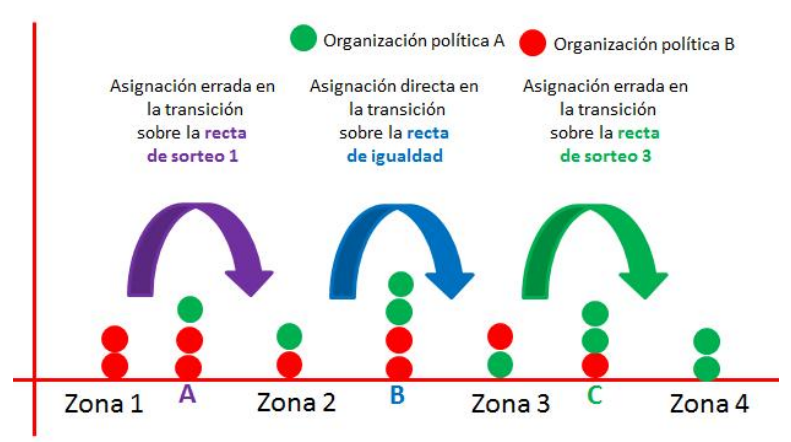

Figura 2. Asignaciones sobre las rectas dependientes $1 / 2$, 1,2 .

Finalmente cuando la cantidad de votos del Partido A sea igual a la del B, la asignación será la siguiente (Tabla 7):

Tabla 7. Asignación de dos votos a dos organizaciones con votos en relación $1 / 1$.

\begin{tabular}{|c|cccc|}
\hline 4 & & Partido A & Partido B & Partido C \\
\cline { 2 - 5 } 5 & Votos & 12000,00 & 12000,00 & 0,00 \\
6 & Asambleístas & & & \\
7 & 2 & $\mathbf{1 2 0 0 0 , 0 0}$ & $\mathbf{1 2 0 0 0 , 0 0}$ & 0,00 \\
\hline
\end{tabular}

Esta situación se puede resolver sin necesidad del método de D'Hondt, con una simple asignación. 


\subsection{Tercera exigencia, para tres escaños y dos organizaciones}

A medida que incrementan los escaños a repartir (entre dos organizaciones políticas) aparecen más rectas de sorteo y zonas de estudio.

Para el caso de tres escaños se presentan las rectas con relaciones o pendientes $1 / 3,1 / 1,3 / 1$, todas simétricas con respecto a la recta identidad en las que debe recurrirse a sorteos para definir el último escaño.

$$
\begin{gathered}
\text { Partido } B=(1 / 3) \cdot \text { Partido } A \\
\text { Partido } B=\text { Partido } A \\
\text { Partido } B=(3) \cdot \text { Partido } A
\end{gathered}
$$

En las Tablas 8, 9 y 10 se muestran las asignaciones con sorteo para las rectas con pendiente $1 / 3,1 / 1$ y $3 / 1$ respectivamente.

Tabla 8. Asignación de tres escaños a dos organizaciones con votos en relación $1 / 3$.

\begin{tabular}{|c|cccc|}
\hline 4 & & Partido A & Partido B & Partido C \\
\cline { 2 - 5 } 5 & Votos & 21000,00 & 7000,00 & 0,00 \\
6 & Asambleístas & & & \\
7 & 1 & $\mathbf{2 1 0 0 0 , 0 0}$ & 7000,00 & 0,00 \\
8 & 1 & $\mathbf{1 0 5 0 0 , 0 0}$ & 7000,00 & 0,00 \\
9 & 2 & $\mathbf{7 0 0 0 , 0 0}$ & $\mathbf{7 0 0 0 , 0 0}$ & 0,00 \\
\hline
\end{tabular}

Tabla 9. Asignación de tres escaños a dos organizaciones con votos en relación $1 / 1$.

\begin{tabular}{|c|cccc|}
\hline 4 & & Partido A & Partido B & Partido C \\
\cline { 2 - 5 } 5 & Votos & 45000,00 & 45000,00 & 0,00 \\
6 & Asambleístas & & & \\
7 & 2 & $\mathbf{4 5 0 0 0 , 0 0}$ & $\mathbf{4 5 0 0 0 , 0 0}$ & 0,00 \\
8 & 2 & $\mathbf{2 2 5 0 0 , 0 0}$ & $\mathbf{2 2 5 0 0 , 0 0}$ & 0,00 \\
\hline
\end{tabular}

Tabla 10. Asignación de tres escaños a dos organizaciones con votos en relación $3 / 1$.

\begin{tabular}{|c|cccc|}
\hline 4 & & Partido A & Partido B & Partido C \\
\cline { 2 - 5 } 5 & Votos & 10000,00 & 30000,00 & 0,40 \\
6 & Asambleístas & & & \\
7 & 1 & 10000,00 & $\mathbf{3 0 0 0 0 , 0 0}$ & 0,40 \\
8 & 1 & 10000,00 & $\mathbf{1 5 0 0 0 , 0 0}$ & 0,40 \\
9 & 2 & $\mathbf{1 0 0 0 0 , 0 0}$ & $\mathbf{1 0 0 0 0 , 0 0}$ & 0,40 \\
\hline
\end{tabular}

En la Figura 3 se muestran la región en dos dimensiones con las rectas de sorteo en el caso de tres escaños a repartir entre dos organizaciones.

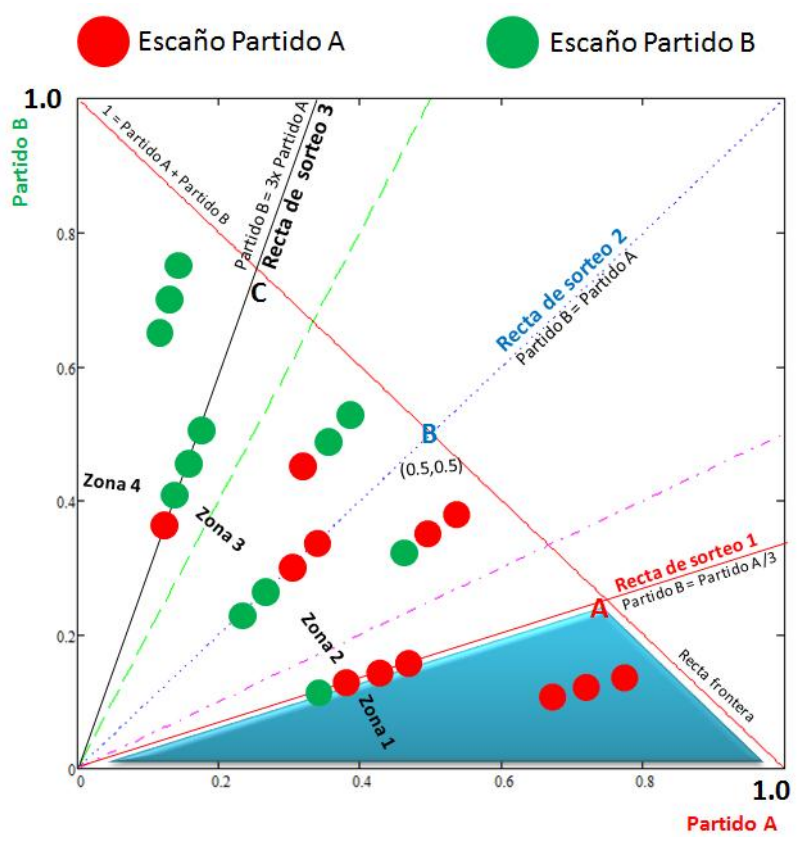

Figura 3. Mapa de asignación de tres escaños a dos organizaciones.

\subsection{Otros escenarios}

El caso de tres organizaciones y dos escaños a repartir, requiere el manejo de regiones contenidas en planos en el espacio tridimensional, todas con coordenadas positivas, como las muestra la Figura 4.

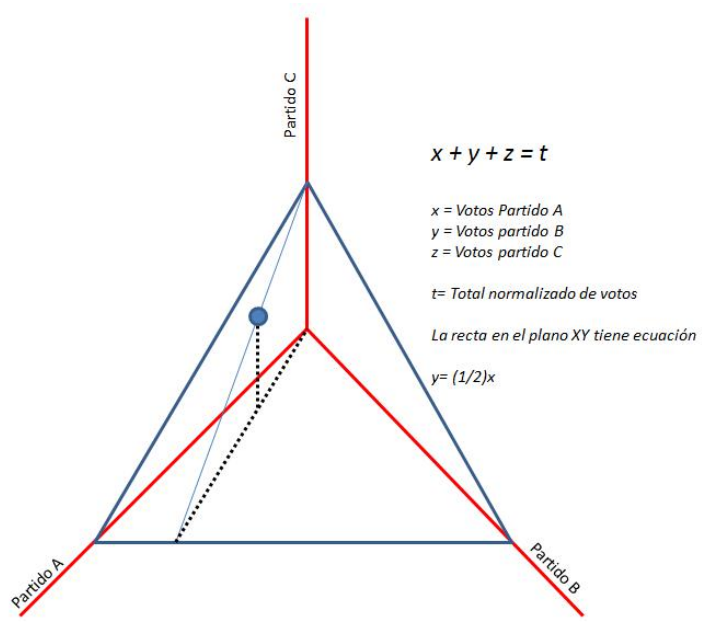

Figura 4. Asignación de dos escaños a tres organizaciones.

Asumiendo que los votos de las organizaciones A y B están en relación 1/2 las ecuaciones relacionadas son:

$$
n_{2}=(1 / 2) n_{1}
$$

$$
n_{1}+n_{2}+n_{3}=1 \geq n_{1}+(1 / 2) n_{1}+n_{3}=1
$$




$$
\begin{gathered}
(3 / 2) n_{1}=-n_{3}+1 \\
n_{1}=-(2 / 3) n_{3}+2 / 3 \\
\sin _{3}=0,4=>n_{1}=(-0,8+2) / 3=0,4 \\
n_{2}=(1 / 2) n_{1}=(1 / 2) \cdot 0,4=0,2
\end{gathered}
$$

Para los ejemplos se considera valores normalizados (todos los valores divididos entre el total votos).

Tabla 11. Asignación de tres escaños a tres organizaciones; las dos primeras con votos en relación $1 / 2$.

\begin{tabular}{|l|cccc|}
\hline 4 & & Partido A & Partido B & Partido C \\
\cline { 2 - 5 } 5 & Votos & 0,40 & 0,20 & 0,40 \\
6 & Asambleístas & & & \\
7 & 2 & $\mathbf{0 , 4 0}$ & 0,20 & $\mathbf{0 , 4 0}$ \\
8 & 3 & $\mathbf{0 , 2 0}$ & $\mathbf{0 , 2 0}$ & $\mathbf{0 , 2 0}$ \\
\hline
\end{tabular}

Lo mostrado anteriormente permite encontrar las diferentes combinaciones en las cuales se va a requerir de sorteo para asignar los escaños. En la Figura 4 se induce las posibles combinaciones que se manifiestan en el lugar geométrico del plano $3 D$ y a medida que aumente los escaños y organizaciones la complejidad en la representación irá en aumento.

\section{Resultados y conclusiones}

Ejemplos de asignación de dos escaños por distrito para los casos de sorteo luego de aplicar D'Hondt.

La Tabla 12 muestra la asignación, por sorteo, de tres escaños $(50 \%)$ para la Organización B, mientras que la Tabla 13 muestra la asignación, por sorteo, de la totalidad $(100 \%)$ de escaños a la Organización A.

En otro orden de ideas, si la organización A logra un voto adicional en cada caso, se alzará con el $100 \%$ de escaños, de acuerdo al método de D'Hondt. En el caso que la Organización A obtenga un voto menos en cada distrito le corresponderían 3 escaños igual al $50 \%$.

El método de D'Hondt, a la vez que se puede interpretar como un apoyo a las organizaciones mayoritarias, es considerado como un impulsador a la unión de organizaciones con pocos votos lo que puede denominarse cooperativismo político [5].

En el caso que todas las organizaciones, para fortalecerse, se agrupen en dos polos: Organización A y

\begin{tabular}{|c|c|c|}
\hline \multicolumn{3}{|c|}{ Distrito 1} \\
\hline $\begin{array}{c}\text { Votos } \\
\text { Primer escaño } \\
\text { Segundo }\end{array}$ & $\begin{array}{c}\text { Organización A } \\
100 \\
1 \\
\quad * \text { El segundo }\end{array}$ & $\begin{array}{l}\text { Organización B } \\
50 \\
1^{*} \\
\text { ue por sorteo }\end{array}$ \\
\hline \multicolumn{3}{|c|}{ Distrito 2} \\
\hline $\begin{array}{l}\text { Votos } \\
\text { Primer escaño } \\
\text { Segundo }\end{array}$ & $\begin{array}{c}\text { Organización A } \\
20 \\
1 \\
\text { *El segundo }\end{array}$ & $\begin{array}{c}\text { Organización C } \\
10 \\
1^{*} \\
\text { fue por sorteo }\end{array}$ \\
\hline \multicolumn{3}{|c|}{ Distrito 3} \\
\hline $\begin{array}{c}\text { Votos } \\
\text { Primer escaño } \\
\text { Segundo }\end{array}$ & $\begin{array}{c}\text { Organización A } \\
10 \\
1 \\
\text { *El segundo }\end{array}$ & $\begin{array}{c}\text { Organización D } \\
5 \\
1^{*} \\
\text { ue por sorteo }\end{array}$ \\
\hline
\end{tabular}
Organización B, como ha ocurrido en muchos países,
Tabla 12. Asignación por sorteo. Todos los sorteados corresponden a las organizaciones B, C y D.

Tabla 13. Asignación extrema por sorteo. Todos los sorteados corresponden a la organización A.

\begin{tabular}{ccc}
\hline & Distrito 1 \\
\hline Organización A & Organización B \\
Votos & 100 & 50 \\
Primer escaño & 1 & \\
Segundo & $1^{*}$ & \\
& $*$ El segundo fue por sorteo
\end{tabular}

\begin{tabular}{ccc}
\hline & Distrito 2 \\
\hline & Organización A & Organización C \\
Votos & 20 & 10 \\
Primer escaño & 1 & \\
Segundo & $1^{*}$ & \\
& $*$ El segundo fue por sorteo \\
\hline & Distrito 3 \\
\hline Ootos & 10 & 5 \\
Primer escaño & 1 & Organización A \\
Segundo & $1^{*}$ & \\
& $*$ El segundo fue por sorteo \\
\hline
\end{tabular}

deduciré la fórmula inédita que relacionan las cantidades de votos para que cada una obtenga la mitad de escaños $(50 \%)$ o una distribución alterna, sin considerar el caso extremo de igual cantidad de votos que se dilucidaría en base a sorteos. Esta fórmula de bolsillo [6] rápidamente le entregará los rangos de valores y otros requerimientos, como se verá en los ejercicios, sin recurrir al simulador y permitiría hacer análisis con una simple calculadora de mano. 


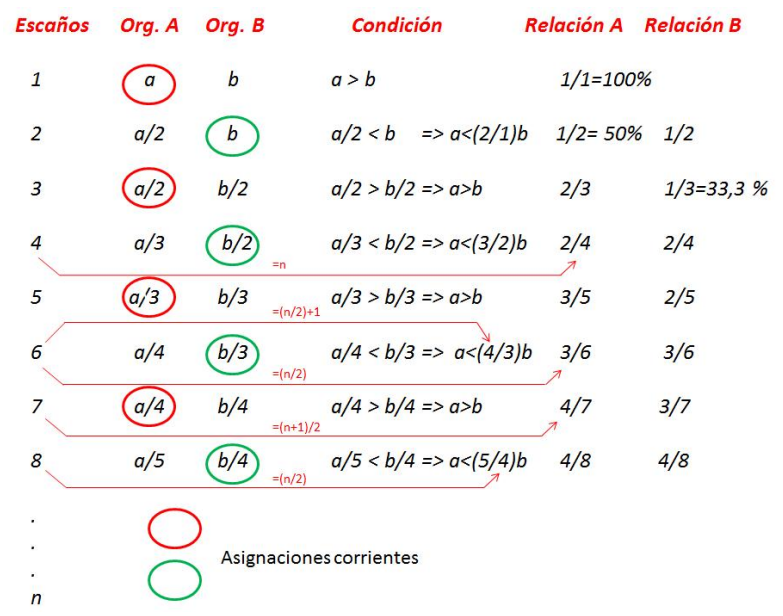

Figura 5. Asignación máxima de $n$ escaños entre dos únicas organizaciones participantes.

\section{Deducción de fórmulas de relación óptima de cantidad de votos en dos organizaciones}

Como primer paso se determinará la condición para conseguir el máximo de escaños, de un total de $n$, para las dos únicas organizaciones participantes en un proceso eleccionario en función de $n$ tomando como base la Figura 5.

$$
b<a<\frac{\left(\frac{n}{2}\right)+1}{\left(\frac{n}{2}\right)} b=\frac{n+2}{n} b \quad \text { si } n \text { es par }
$$

Donde:

- a cantidad de votos de la organización $A$.

- $b$ cantidad de votos de la organización $B$.

Con esta condición mantiene el $50 \%$ de los escaños.

\section{Porcentaje de la organización A}

$$
\begin{gathered}
\text { Si } n \text { es par } \%=\frac{\frac{n}{2}}{n} \cdot 100=50 \% \\
\text { Si } n \text { es impar } \%=\frac{\left(\frac{n+1}{2}\right)}{n} \cdot 100=\ldots \\
\ldots=\frac{n+1}{2 n} \cdot 100
\end{gathered}
$$

Se observa que cuando $n$ tiende a infinito el porcentaje tiende a $50 \%$.

\section{Ejercicio 1}

La última encuesta sugiere que la organización A considerada primera fuerza va a obtener 1520 votos. La segunda fuerza quiere saber cuántos votos debe obtener, como mínimo, para lograr el $50 \%$ de los 8 escaños que se van a repartir.

Tomando como base la Ecuación 15

$$
\frac{(n+2)}{n}=\frac{(8+2)}{8}=1,25
$$

esto implica que $b$ debe satisfacer:

$$
\begin{gathered}
a<1,25 b=>b>a / 1,25= \\
1520 / 1,25=1216
\end{gathered}
$$

Lo que implica:

1. Con menos de 1216 obtiene menos del $50 \%$

2. Con 1216 cae en zona de sorteo

3. Con más de 1216 y menos de 1520 obtiene $50 \%$

Tabla 14. Asignación en vecindad del $50 \%$.

\begin{tabular}{|c|cccc|}
\hline 4 & & Partido A & Partido B & Partido C \\
\cline { 2 - 5 } 5 & Votos & 1520,00 & 1215,00 & 0,00 \\
6 & Asambleístas & & & \\
7 & 1 & $\mathbf{1 5 2 0 , 0 0}$ & 1215,00 & 0,00 \\
8 & 1 & 760,00 & $\mathbf{1 2 1 5 , 0 0}$ & 0,00 \\
9 & 1 & $\mathbf{7 6 0 , 0 0}$ & 607,50 & 0,00 \\
10 & 1 & 506,67 & $\mathbf{6 0 7 , 5 0}$ & 0,00 \\
11 & 1 & $\mathbf{5 0 6 , 6 7}$ & 405,00 & 0,00 \\
12 & 1 & 380,00 & $\mathbf{4 0 5 , 0 0}$ & 0,00 \\
13 & 1 & $\mathbf{3 8 0 , 0 0}$ & 303,75 & 0,00 \\
14 & 1 & $\mathbf{3 0 4 , 0 0}$ & 303,75 & 0,00 \\
\hline
\end{tabular}

\begin{tabular}{|c|cccc|}
\hline 4 & & Partido A & Partido B & Partido C \\
\cline { 2 - 5 } 5 & Votos & 1520,00 & 1216,00 & 0,00 \\
6 & Asambleístas & & & \\
7 & 1 & $\mathbf{1 5 2 0 , 0 0}$ & 1216,00 & 0,00 \\
8 & 1 & 760,00 & $\mathbf{1 2 1 6 , 0 0}$ & 0,00 \\
9 & 1 & $\mathbf{7 6 0 , 0 0}$ & 608,00 & 0,00 \\
10 & 1 & 506,67 & $\mathbf{6 0 8 , 0 0}$ & 0,00 \\
11 & 1 & $\mathbf{5 0 6 , 6 7}$ & 405,33 & 0,00 \\
12 & 1 & 380,00 & $\mathbf{4 0 5 , 3 3}$ & 0,00 \\
13 & 1 & $\mathbf{3 8 0 , 0 0}$ & 304,00 & 0,00 \\
14 & 1 & $\mathbf{3 0 4 , 0 0}$ & $\mathbf{3 0 4 , 0 0}$ & 0,00 \\
\hline
\end{tabular}

\begin{tabular}{|c|cccc|}
\hline 4 & & Partido A & Partido B & Partido C \\
\cline { 2 - 5 } 5 & Votos & 1520,00 & 1217,00 & 0,00 \\
6 & Asambleístas & & & \\
7 & 1 & $\mathbf{1 5 2 0 , 0 0}$ & 1217,00 & 0,00 \\
8 & 1 & 760,00 & $\mathbf{1 2 1 7 , 0 0}$ & 0,00 \\
9 & 1 & $\mathbf{7 6 0 , 0 0}$ & 608,50 & 0,00 \\
10 & 1 & 506,67 & $\mathbf{6 0 8 , 5 0}$ & 0,00 \\
11 & 1 & $\mathbf{5 0 6 , 6 7}$ & 405,67 & 0,00 \\
12 & 1 & 380,00 & $\mathbf{4 0 5 , 6 7}$ & 0,00 \\
13 & 1 & $\mathbf{3 8 0 , 0 0}$ & 304,25 & 0,00 \\
14 & 1 & 304,00 & $\mathbf{3 0 4 , 2 5}$ & 0,00 \\
\hline
\end{tabular}




\section{Ejercicio 2}

La circunscripción tiene un padrón electoral de 2200 electores y ocho escaños para asambleístas. Si la organización A estima obtener 1520 votos. ¿Qué es lo máximo que puede aspirar la organización $\mathrm{B}$ ?

La organización $\mathrm{B}$ puede obtener un máximo de $2200-1520=680$ votos lo que corresponde a dos escaños según el resultado de la Tabla 15.

Tabla 15. Asignación de escaños a dos organizaciones con 1520 y 680 votos

\begin{tabular}{|c|cccc|}
\hline 4 & & Partido A & Partido B & Partido C \\
\cline { 2 - 5 } 5 & Votos & 1520,00 & 680,00 & 0,00 \\
6 & Asambleístas & & & \\
7 & 1 & $\mathbf{1 5 2 0 , 0 0}$ & 680,00 & 0,00 \\
8 & 1 & $\mathbf{7 6 0 , 0 0}$ & 680,00 & 0,00 \\
9 & 1 & 506,67 & $\mathbf{6 8 0 , 0 0}$ & 0,00 \\
10 & 1 & $\mathbf{5 0 6 , 6 7}$ & 340,00 & 0,00 \\
11 & 1 & $\mathbf{3 8 0 , 0 0}$ & 340,00 & 0,00 \\
12 & 1 & 304,00 & $\mathbf{3 4 0 , 0 0}$ & 0,00 \\
13 & 1 & $\mathbf{3 0 4 , 0 0}$ & 226,67 & 0,00 \\
14 & 1 & $\mathbf{2 5 3 , 3 3}$ & 226,57 & 0,00 \\
\hline
\end{tabular}

\section{Ejercicio 3}

El consejo electoral de una región está buscando asegurar que en una asignación de escaños a las dos únicas organizaciones políticas, la más fuerte no sobrepase del $57,14 \%$ en un universo de 7200 electores. ¿Cuántos escaños se deben repartir y cuántos votos debe obtener cada organización?

Como el porcentaje está por encima del $50 \%$ (que sucede cuando $n$ es par), debe tomarse la Ecuación 3 para $n$ impar. El porcentaje se determina con la fórmula $\frac{(n+1)}{2 n}=0,5714=>n=7$ escaños. Con la mínima diferencia de votos entre ellos se obtienen los necesarios para la organización A $(72000 / 2)+1=3601$.

Tabla 16. Asignación con condición de 57,14\%

\begin{tabular}{|c|cccc|}
\hline 4 & & Partido A & Partido B & Partido C \\
\cline { 2 - 5 } 5 & Votos & 3601,00 & 3599,00 & 0,00 \\
6 & Asambleístas & & & \\
7 & 1 & $\mathbf{3 6 0 1 , 0 0}$ & 3599,00 & 0,00 \\
8 & 1 & 1800,50 & $\mathbf{3 5 9 9 , 0 0}$ & 0,00 \\
9 & 1 & $\mathbf{1 8 0 0 , 5 0}$ & 1799,50 & 0,00 \\
10 & 1 & 1200,33 & $\mathbf{1 7 9 9 , 5 0}$ & 0,00 \\
11 & 1 & $\mathbf{1 2 0 0 , 3 3}$ & 1199,67 & 0,00 \\
12 & 1 & 900,25 & $\mathbf{1 1 9 9 , 6 7}$ & 0,00 \\
13 & 1 & $\mathbf{9 0 0 , 2 5}$ & 899,75 & 0,00 \\
\hline
\end{tabular}

\section{Ejercicio 4}

En una provincia se van a repartir cuatro escaños entre dos organizaciones políticas mayoritarias. Se pide definir los rangos de votos que permitan que cada organización obtenga el $50 \%$ de escaños.

Considerando la Ecuación 15 y $n=4=>b<a<$ $\frac{\left(\frac{n}{2}\right)+1}{\left(\frac{n}{2}\right)} b=\frac{n+2}{n} b=>b<a<1,5 b$ lo cual lleva a las ecuaciones siguientes $a+b=1 \& a=1,5 b=>1,5 b+b=$ $1=>2,5 b=1=>b=0,4=>b=40 \%$ y $a=60 \%$

Tabla 17. Asignación de escaños a dos organizaciones con $60 \%$ y $40 \%$ de votos.

\begin{tabular}{|c|cccc|}
\hline 4 & & Partido A & Partido B & Partido C \\
\cline { 2 - 5 } 5 & Votos & 21000,00 & 14001,00 & 0,00 \\
6 & Asambleístas & & & \\
7 & 1 & $\mathbf{2 1 0 0 0 , 0 0}$ & 14001,00 & 0,00 \\
8 & 1 & 10500,00 & $\mathbf{1 4 0 0 1 , 0 0}$ & 0,00 \\
9 & 1 & $\mathbf{1 0 5 0 0 , 0 0}$ & 7000,50 & 0,00 \\
10 & 1 & 7000,00 & $\mathbf{7 0 0 0 , 5 0}$ & 0,00 \\
\hline
\end{tabular}

\section{Conclusiones}

La conclusión principal en este trabajo es que se pueden obtener fórmulas [7] para fijar condiciones previas a la corrida con el simulador o aplicación manual del método de D'Hondt, lo cual conlleva a mayor precisión y rapidez en el planteamiento de escenarios [8]. Partiendo de la premisa que el método llegó para quedarse entonces es fundamental abocarse a preparar un conjunto de fórmulas para el planteamiento científico de alianzas o estrategias. Aunque el punto de partida está basado en dos organizaciones es necesario y en eso seguiré trabajando para un poco más de organizaciones, pero también reforzar la teoría para el caso de dos organizaciones que de alguna manera sugiere el método de D'Hondt: El cooperativismo político.

De gran ayuda resultaron los conocimientos de geometría analítica del plano y del espacio [9], el manejo de series, teoría de inducción matemática y el simulador que preparé en Visual Basic para Aplicaciones (VBA) sobre la plataforma Excel para Windows [10], para probar escenarios. Este simulador puede ser bajado, sin condiciones desde http://bolivarlojan.blogspot. $\mathrm{com} / \mathrm{p} /$ dhondtel-cooperativismo-politico.html

\section{Referencias}

[1] Ecuador Times, "Corte Constitucional resuelve Código de la Democracia." [Online]. Available: http://www.ecuadortimes.net/es/2012/10/18/ corte-constitucional-resuelve-codigo-democracia/

[2] F. Girón González-Torre and J. M. B. Herránz, "Las matemáticas de los sistemas electorales," Revista Real Academia de Ciencias Exactas 
Físicas y Naturales, vol. 101, no. 1, pp. 21--33, 2007. [Online]. Available: http://www.rac.es/ ficheros/doc/00470.pdf

[3] C. Luna, "La Ley D'Hondt como nunca te la habían explicado antes." [Online]. Available: http://blog.pseudolog.com/article/la-ley-d-hont

[4] S/a, "Cómo funciona la Ley D'Hondt." [Online]. Available: http: //www.microsiervos.com/archivo/mundoreal/ como-funciona-la-ley-d-hondt.html

[5] R. Coopecas, "Objetivos del cooperativismo," 2011. [Online]. Available: http://asesoria.obolog. com/objetivos-cooperativismo- 1243572

[6] R. Chang Motta, Los sistemas electorales. [Online]. Available: http://biblio.juridicas.unam. $\mathrm{mx} /$ libros $/ 2 / 635 / 18 . p d f$
[7] F. Robles, "Fórmulas electorales, proporcionalidad y bipartidismo modificado: Los casos de Colombia y Venezuela," Papel Político, no. 2, pp. 45--62, 1995. [Online]. Available: http://javeriana.edu.co/ Facultades/politicas/publicaciones/documents/ 3.FORMULASELECTORALES.pdf

[8] X. Arnau, 'D'Hondt Calculator, la app que calcula el reparto de escaños," 2011. [Online]. Available: http://www.yorokobu.es/ dhondt-calculator-la-app-que-calcula-el-reparto-de

[9] Geoan, "Geometría Analítica en el plano," 2008. [Online]. Available: http://www.geoan.com/

[10] Microsoft Corporation, "Introducción a VBA en Excel 2010," 2010. [Online]. Available: http://msdn.microsoft.com/es-es/library/ office $/$ ee $814737 \% 28 \mathrm{v}=$ office. $14 \% 29$.aspx 